\title{
A New Model for Intelligent Component Based System Analysis: Intelligent Unit
}

\author{
Zhou Yuan* $^{*}$
}

Guangdong Polytechnic Normal University, Guangzhou, 510665 P.R. China

\begin{abstract}
Many complex models and approaches are utilized for the analysis of resources, scales and scheduler of software systems. The challenge is still present to accurately approximate the resources for Web based applications and intelligent systems softwares. The user requirements and capabilities are continuously altering, such situations produce problems dealing with environments related to intelligent systems. Hence, there is requirement to introduce a standalone model for approximation of Web based systems. This research paper presents an intelligent Unit, a logic system to model a software resource analysis process using intelligent logic, thereby satisfying many conditions of criteria by using Analysis Model, a famous classical algorithmic approach. The research is mostly focused on finding differences between cloud computing and common approaches. The research approach employed improves significance levels which are essential for planning phases.
\end{abstract}

Keywords: Analysis, Cloud Computing, Intelligent Component, Statement.

\section{INTRODUCTION}

The classical approach for developing life cycle models is utilized for Software Systems analysis [1]. There is limitation using these models in terms of technology usage and satisfaction level. The development challenges include predicting the time and resource analysis for Software Systems. The estimates obtained at the initial stages of life cycle are not accurate enough, because lot of details are not available at that time and time span between signing and delivery of the system is of much duration. Recently, the software development cycle is using new approaches and many new trends like intelligent component development, rapid application development and continuous prototyping are being introduced. The experts in this field have designed these new trends in the development of life cycle [2]. These new trends are widely being investigated and it is a challenge to find whether any new trend is suitable to use for a specific task [3]. The estimation for Web based systems is also a challenging task while considering limited availability of resources. The software programmers are interested to find out the time and efforts involved in the successful functioning of Software Systems. Industries and software developers are investigating and using models based on techniques of approximation and iteration. The analysis of Software Systems is very crucial for companies and developers because it produces many benefits such as resource control. The software analysis approach consists of three elements which includes Web resource analysis model, software metric and software analysis tool [4]. Many quantitative software resource

*Address correspondence to this author at the Guangdong Polytechnic Normal University, Guangzhou, 510665, P.R. China;

E-mail:ycz_q@126.com analysis models are in the development phase. The regression approach is extensively being used for these analysis models. These models are based on mathematics, data collection and formation of regression equation.

In this research, an intelligent Unit model is proposed, which can be used for analyzing and providing intelligent algorithms required for developing softwares. The resource analysis models are investigated in Section 2. In Section 3, intelligence based Web development system and its features are discussed. Section 4 elaborates the problems arising in Web resource analysis, Sections 5 and 6 describe the problem statement and the developed software model. The model proposed for analysis is discussed in the next section.

\section{SOFTWARE RESOURCE ANALYSIS MODELS}

There is requirement to find out the total development time of a project from start to end. The program scale is used as a parameter for such resource models. These earlier models do not replicate original models and thus resultantly do not have much accuracy. Software analysis has become a worldwide major concern because of its financial and strategic impact. Fig. (1) shows the Software Resource Analysis approach which is based on Model Based and Non-Logic Models methods [5].

The statistical data analysis as used conventionally is used for Algorithmic models while Non-Logic approaches are comprised of new trends like price to win, Match learning and experts judgment.

\subsection{Algorithmic Models}

Boehm's analysis method, Albert's Function Number and SLIM by Putnam are being used currently and are popular 


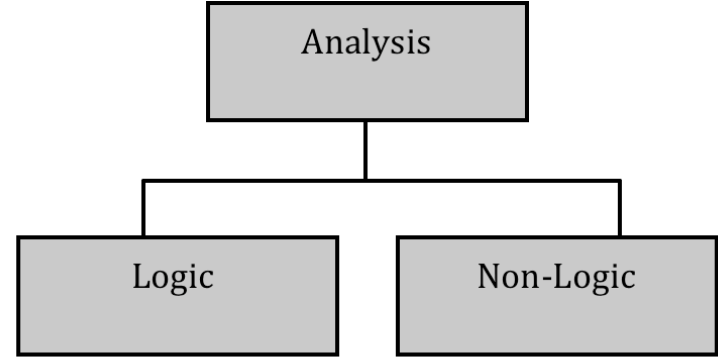

Fig. (1). Software Resource Analysis.

choices among software developers. All these models require certain estimates about specific parameters and attributes including Unit of Content (UOC), complexity interface and total number of user's on screen. Such values estimation is very much likely to be in the initial phases. Recently used approaches for Classification models are presented in Fig. (2). The computation of these development models is a challenging task due to inherited complexity among different attributes. The estimation is not precise and can change during development environment. These limitations present in development model resulted in a new approach called NonLogic method.

\subsection{Non-Logic Models}

Non-Logic models are being used from early 90's. Some new approaches like fuzzy logic, genetic algorithm and artificial neural network are being investigated for software development models, which are based on soft computing.

One of the techniques used for Non-Logic models is Expert Judgment, which is based on experiences of System managers. It can be revealed that expert Judgment provides better approximation as compared to the model based estimates [6]. The decision is taken on considering personnel interests in Thumb Rule Decision, this technique however also has some drawbacks. If Delphi approach is considered, there is no interaction available among developers and it is the responsibility of the Coordinator to take care of all events of the process. Rand Corporation have introduced a new technique called Wide Band Delphi technique, all experts are agreed on benefits of this approach. The decision whether to Buy vs. Make is based on reusing software development components. The System resources are calculated in any company's resource limit which is based on Parkinson's Law. The user's budget play an important role in determining pricing technique as in any business, but when detailed data is not provided it may be the best optimal solution. Watt has introduced the PSP Probe method, which is based on work experiences when future efforts are considered. The team dynamics planning, role defining and development stages are important features when TSP Planning (Team Software Process) is considered.

\section{INTELLIGENCE BASED WEB DEVELOPMENT SYSTEMS AND CHARACTERISTICS}

For Web based Systems, approximations have been carried out for scheduler and resources by experts. Advanced

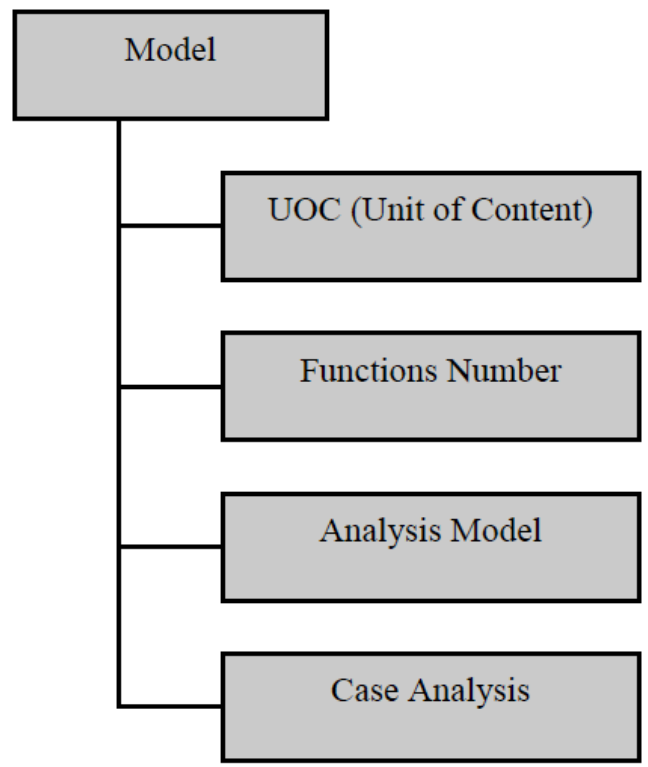

Fig. (2). Software Analysis Classification.

methods like Java applets, hypertext markup language, Java scripts and many others are being extensively investigated for generating software quickly, such techniques beat analysis procedures, resists models and ignore scale matrices. Many questions remained unanswered while using these fast paced software development techniques. The web objects are made functional in quick time frame while using such accelerated techniques.

The emphasis carried out in this research is to use software development with component based, reuse systematic resources and visual technologies [7]. This results in considering fast paced technologies whose purpose is to ensure faster delivery to the market. This scheme is used by companies which run Web based business. The software development is changing at a high pace, despite considering users need, general programs are being developed using intelligent Web development and comprehensive documentation. The expert teams work together for developing iterative and reusable resources for continuous and rapid prototype development methods. For Web based resource estimation, Intelligent analysis using Functional Matrices is being used [8]. Limited resources companies face difficulty in the estimation using Intelligent components.

Web development utilizes intelligent technologies and cloud computing which are different approaches. The challenges experienced in intelligent analysis are precise analysis of scale and timing [7]. The new altered matrices are preferably applied to Web programs like java scripts, shopping carts, and building blocks like Active X control, Cookies and Object model components. The cube roots law which is extensively used for duration analysis equations does not work for Web based development.

\section{INTELLIGENT SOFTWARE DEVELOPMENT}

The iteration and increment developments are important aspects of intelligent software development technique. The 
companies and experts team work collaboratively to enhance improvements in the development cycle [9].

Many advantages are achieved by using this approach which includes evolution development and delivery, timeboxed iterative model and adaptive preparations. It also has increased response and therefore, reacts to any changes abruptly. The intelligent development approach is based on concepts and it is also responsible for collaborations among different experts teams during the whole developmental phase.

\subsection{Manifestos for Intelligent Software Development}

The manifesto for intelligent software development was defined and finalized in 2001. It consists of four statements which are given below:

1) The interaction between individuals for different tools and processes;

2) Complete documentation needed for suitable software;

3) Interaction among users for negotiating contract;

4) For whole time during completion of task, act on a plan and respond to changes.

\subsection{Intelligent Methods and Characteristics}

There are many intelligent approaches available. Most of them emphasize on interaction, process adaptability and team work among different groups of developers throughout the whole process. The huge task is divided into small tasks and long-term planning is not carried out for this approach, thus less effort with planning is required [11]. Iterations are considered for short duration, because they are available for one to four weeks only. For each iterations, developer's teams are responsible for smooth functioning of analysis, design, unit testing, coding and results acceptance. When the iteration is finished, concerned individuals are given a running product, which helps to overcome different challenges and faster adaptation to the abrupt changes. There is extensive use of Intelligent Methods. Some of these methods are as follows:

1) Extreme Programming;

2) Feature-Driven Development;

3) Lean Development;

4) Adaptive Software Development.

\section{NEW MODEL \& INTELLIGENT COMPONENT EVENT}

For the networks, which are not able to develop interaction between each other, an intelligent event component is utilized for overcoming this issue. The connections can be broken down due to many reasons like wearing out, mobility, random installation, changed conditions etc. If nodes are combined, the emission power is increased because nodes are joined collectively. Identical symbol sets are propagated for collective waves in a synchronous order. At the receiving end, summed up signals containing high power are collected. If there are more nodes, then the higher power signals will be

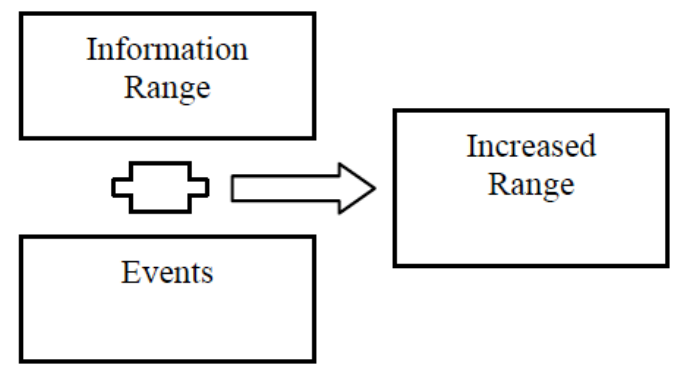

Fig. (3). Increased Range.

transmitted to the physical medium. If the power is high, then the far destinations can also be reached.

This research is focused on issues arising in building multi-platform, MAC sub-platforms and lower platform's transmission information. There is always chance of improvements in interaction among MAC, physical and network platforms. The channel information is transmitted via physical platform to the end network platform. Power and data rate information is sent from network platform to the physical platform.

The dynamical information model is built considering $\mathrm{N}$ nodes, whose distribution occur in a 2-dimensional plane. A graph can be made for $\mathrm{G}(\mathrm{V}, \mathrm{E})$, where $\mathrm{V}=\left\{\mathrm{v}_{1}, \mathrm{v}_{2}, \mathrm{v}_{3}, \cdots\right.$, $\left.\mathrm{v}_{\mathrm{n}}\right\}$ represents finite nodes set and $\mathrm{E}=\left\{\mathrm{e}_{1}, \mathrm{e}_{2}, \mathrm{e}_{3}, \cdots, \mathrm{e}_{\mathrm{n}}\right\}$ indicates Links set. The nodes and link sets are selected randomly. Fixed infrastructure is not available for information networks, therefore these networks work collectively. Each node has information of its own and other's node location; the network is thus dynamic.

The destination location is known by the set of two nodes represented as $\mathrm{V}$-source $=\left\{\mathrm{VS}_{1}, \mathrm{VS}_{2}, \cdots, \mathrm{VSn}\right\}$. A lot of information is sent to $d(i)$ nodes from the $V$-source. Each node $i$ sends a lot of information to destination $d(i)$, which may be presented in $\mathrm{S}$ region. Assume $\mathrm{h}_{\mathrm{ij}}$ represents the gain power between nodes $\mathrm{i}$ and $\mathrm{j}$. All nodes presented are denoted by E-total and they have constraints limitation. The allocated resources in a node are responsible for transmitting and relaying this specific node information. All available nodes are in a fully charged state and have identical resources. For source destination (s-d) pair, distance may be presented between these two nodes; their function is to minimize errors, increase power efficiency and also reduce transmission related delays. The increased event range is depicted in Fig. (3).

The term Atx is assumed as the transmitted power related to the node. It is also assumed that all nodes comprised of identical power.

$A_{r x, j} \leftarrow i$ represents the power received from propagated signal from nodes $\mathrm{i}$ to $\mathrm{j}$. A receiver node power $P_{r x, j} \leftarrow i$ will provide SNR value in the receiver for decoding the information. Power gain is unfairly utilized in collective information transmission approach. At receiving end, $\mathrm{j}$ node can receive collective power from the set of $\mathrm{G}$ nodes.

$$
P_{r x, j} \leftarrow G=\sum_{i \in G} P_{r x, j} \leftarrow i
$$


Table 1. Key attributes with rating levels.

\begin{tabular}{|c|c|c|c|c|c|c|}
\hline Words & $\boldsymbol{d}_{\mathbf{1}}$ & $\boldsymbol{d}_{\mathbf{2}}$ & $\boldsymbol{d}_{\mathbf{3}}$ & $\boldsymbol{d}_{\mathbf{4}}$ & $\cdots$ & $\boldsymbol{d}_{\boldsymbol{n}}$ \\
\hline \hline & 2 & 2 & 3 & 2 & 0 \\
\hline & 1 & 0 & 1 & 2 & 0 \\
\hline & 0 & 1 & 4 & 0 & 0 \\
\hline & 0 & 0 & 0 & 0 & 0 \\
\hline
\end{tabular}

A path loss model is considered as a physical platform in developing information system. The difference between transmitted and received power can be found out by Path loss model. Path losses occur during this transmission and deterioration is caused by free space, reflection, scattering and diffraction phenomenon. Path losses can be represented as follows:

$$
L=10 n \log _{10}(d)+C
$$

where L depicts the path loss expressed in decibels, $n$ represents the exponent path loss, $d$ is the distance between the transmission and the receiving end measured in meters, and $\mathrm{C}$ is a constant representing losses in the system.

The losses in radio and event can be computed considering path loss written as follows:

$$
L=20 \log _{10(4 \pi d \mid \lambda)}
$$

where $\mathrm{L}$ denotes the path loss in decibels, $\lambda$ represents the wavelength and $\mathrm{d}$ depicts the transmitter-receiver distance which is measured in same units as the wavelength. Path loss expressed in radial fading $1 / \gamma_{i, j}^{\beta}$, while the maximum distance computed for ideal complete information transmission is $\gamma_{t h}^{\beta}=\frac{P_{t x}}{P_{t h}}$. It is supposed that certain resources $E_{t x}$ are utilized for an event working. There are three sections in hybrid combination: (a) single destination (b) signal source and (c) set of potential nodes B. If interference is present between two nodes, then frame data is lost. If there is partial collision, still they are assumed collided. A mechanism exists which monitors all success and failure data information. The fixed infrastructure is available and the network is considered dynamic.

\section{INTELLIGENT MODEL BASED WEB DEVELOP- MENT}

A programmed plan is essential for system development when considering predictive methods. While adaptive techniques look for checking present suitable conditions and finding an acceptable effective solution at each stage of the system development [10]. The Web analysis uses multiple Web objects and multiple attribute approaches for dealing with intelligent software systems. An exact and specific interaction set in intelligent software and attribute is due to the scale factors and resource drivers. The algorithms used for intelligent system development also produce certain con- straints and limitations on prediction approximations. The identification of attributes relating to intelligent system is guaranteed while considering inbuilt issues. The human/people factor has been given more priority as compared to the process factor. The intelligent unit is suggested in this research for Web based models, because it can adapt to and add new features to the already existing analysis models based on Web MO [7].

The prediction must be ensured when considering various components and formulation process. Considering resource analysis, people are not considered subject to approximations, because it is very challenging to predict. The intelligent systems are subjected to many disturbances factors, uncertainties, risks, emerging requirements and complicated issues. The cloud computing in the intelligent environment also requires mapping for qualitative and quantitative values set. The mapping in intelligent system is ensured by key attributes responsible for intelligent system development. This is shown in Table $\mathbf{1}$ with corresponding rating levels.

This rating specifies a measurement ranking, which informs about quality of product and completion time.

\subsection{Scale Analysis}

High risk analysis is routinely carried out and an effort model is required for Web based system because of their tiny size. The fields of Web related technologies are making progress rapidly. New tools and approaches update information very abruptly and old information becomes obsolete very quickly. The Halsted Equation is used for computing program's length which relies on operands and operators. This scale measure does not depend on any language and it is written as follows:

$$
S=\left(W_{1}+W_{2}\right) \cdot \log _{2}\left(w_{1}+w_{2}\right)
$$

where $\mathrm{W}_{1}$ is the approximation of total Web Operand;

$\mathrm{W}_{2}$ represents the occurrences of total Web Operator estimator; tor;

$\mathrm{W}_{1}=$ total number of specific Web Operands approxima-

$\mathrm{W}_{1}=$ total number of unique Web Operators approximator.

Web objects can be used for scale analysis of the Web development [7]. The scale matrices are used for computation of scales for Web based applications. 


\section{CONCLUSION}

The market trends and realizations exert clients to use intelligent software approach for cloud computing of short duration. Sometimes, the results are not up to standard and they lack accuracy, reliability and cannot meet the set criteria. These challenges and issues are difficult to avoid in such circumstances.

This research has focused on identifying issues arisen in Web developments considering intelligent software development. This identification is obtained by data analysis and intelligent software development approaches. Usually the developers have no previous information to estimate parameters required for Web development procedures. The intelligent systems are relatively new and developers are still depending on estimations. The intelligent features try to make analysis less efficient when time and resources are taken into account. For overcoming this issue, this research has proposed an intelligent Unit model, which does not depend on expert's estimation but it provides more reliable analysis based on Web features and intelligent system developments.

\section{CONFLICT OF INTEREST}

The author confirms that this article content has no conflict of interest.

\section{ACKNOWLEDGEMENTS}

Declared none.

\section{REFERENCES}

[1] A. Cerpa, J. Elson, D. Estrin, L. Girod, M. Hamilton, and J. Zhao "Habitat monitoring: application driver for wireless communications technology," In: ACM SIGCOMM Computer Communication Review - Workshop on Data Communication in Latin America and the Caribbean, vol. 31, no. 2, 2001, pp. 20-41.

[2] K.S. Arefin and E. Ahmed, "Cross-layer design of wireless networking for parallel loading of access points (PLAP)," In: $10^{\text {th }}$ International Conference on Computer and Information Technology, 2007, pp. 1-5.

[3] M.A. Mehr, "Design and implementation a new information utilization rate classification algorithm using genetic algorithm for semantic networks," Engineering and Technology, vol. 53, no. 2, 2011, pp. 430-433.

[4] R. Xu, I. Donald and C. Wunsch, "Classification," IEEE Press, New Jersey, 2009.

[5] R. Hu, "Channel access controlling in semantic network using smart grid system," Applied Mathematics \& Information Sciences, vol. 11, pp. 813-820, 2012.

[6] S. Jin, M. Zhou and A.S. Wu, "Sensor network optimization using a genetic algorithm," Direct, vol. 4, pp. 1-6, 2001.

[7] R. Hu, H. Hu, and H. Xu, "Big data-based intrusion behavior discovery in sensor networks," Journal of Investigative Medicine, vol. 63, no. 8, pp. S55-S55, 2015-12.

[8] H. Chan and A. Perrig, "ACE: an emergent algorithm for highly uniform component formation," Semantic Networks, vol. 2920, pp. 55-67, 2004.

[9] R. Hu, "Stability analyzing of semantic network service via data stream methods", Applied Mathematics \& Information Sciences, vol. 11, pp. 793-798, 2012.

[10] I. Gupta, D. Riordan and S. Sampalli, "Component-Head Election Using Fuzzy Logic for Semantic networks," In: $3^{\text {rd }}$ Annual Communication Networks and Services Research Conference (CNSR'05), Halifax, 2005, pp. 255-260.

[11] R. Hu, H. Hu, and Z.H. Xiao, "Research on searching method of sensor network component based on demand concept set," Journal of Investigative Medicine, vol. 63, no. 8, pp. S55-S55, 2015-12.

(C) Zhou Yuan; Licensee Bentham Open.

This is an open access article licensed under the terms of the Creative Commons Attribution Non-Commercial License (http://creativecommons.org/licenses/by$\mathrm{nc} / 4.0 /$ ) which permits unrestricted, non-commercial use, distribution and reproduction in any medium, provided the work is properly cited. 